\title{
DESIGN DE UMA CADEIRA DE RODAS INTELIGENTE
}

\author{
Rodrigo Antonio Marques Braga Dr. \\ Universidade Federal de Santa Catarina UFSC \\ rodrigo.braga@ufsc.br \\ Ana Veronica Pazmino Dra. \\ Universidade Federal de Santa Catarina UFSC \\ ana.veronica@ufsc.br \\ Ivan Medeiros Msc. \\ Universidade Federal de Santa Catarina UFSC \\ ivan.medeiros@ufsc.br \\ Matheus Alberto Ambrosi (graduando) \\ Universidade Federal de Santa Catarina UFSC \\ matheusalbertoambrosi@gmail.com
}

\begin{abstract}
Resumo: $\mathrm{O}$ artigo apresenta um resumo da fase de projeto informacional para o desenvolvimento multidisciplinar de uma cadeira de rodas inteligente com abordagem na automação e no design universal. É mostrada a fundamentação teórica de cadeiras de rodas inteligentes, design acessível, design universal e ergonomia. Finalmente o trabalho mostra os requisitos tecnológicos, princípios do design universal para o desenvolvimento de uma cadeira de rodas inteligente, o método espiral de projeto adotado para o desenvolvimento e um resultado da modelagem preliminar de uma alternativa de solução, assim como os componentes eletrônicos necessários para a adequação do produto ao maior número de usuários.
\end{abstract}

Palavras-chave: design de produto, cadeira de rodas, design universal

Abstract: The article presents a summary of the informational phase project to develop a multidisciplinary intelligent wheelchair to approach automation and universal design. The theoretical foundation of intelligent wheelchair accessible design, universal design and ergonomic chairs is shown. Finally, the work shows the technological requirements, principles of universal design to develop a intelligent wheelchair, the spiral design method adopted for the development and outcome of the preliminary modeling of an alternative solution, as well as the electronics needed to the suitability of the product to more users.

Keywords: product design, wheelchair accessible, universal design 


\section{INTRODUÇÃO}

Deficiências físicas são frequentemente causadas por acidentes, exposição a produtos químicos, drogas e doenças como esclerose múltipla e paralisia cerebral. Algumas condições médicas podem fazer com que pacientes tenham um controle limitado de alguns músculos dos braços, pernas e rosto, afetando sua mobilidade. A generalização de conceitos para tratar e assistir essas incapacidades ainda não foi alcançada. Geralmente, cada paciente tem uma combinação diferente de sintomas que requerem diferentes tipos de estratégias para ameniza-los.

Além disso, o envelhecimento populacional já é um fenômeno mundial. Segundo o IBGE "A população de idosos representa um contingente de quase 15 milhões de pessoas com 60 anos ou mais de idade ( $8,6 \%$ da população brasileira). Nos próximos 20 anos, a população idosa do Brasil poderá ultrapassar os 30 milhões de pessoas e deverá representar quase $13 \%$ da população ao final deste período". 0 envelhecimento é biologicamente normal e evolui progressivamente, e não se dá, necessariamente, em paralelo ao avanço da idade cronológica. Com isso, a longo prazo, o idoso acaba sendo forçado a modificar sua rotina e hábitos de vida, reduzindo seu desempenho físico e como consequência suas habilidades motoras. A dificuldade em realizar as atividades diárias aumenta gradativamente, atrapalhando a manutenção de um estilo de vida saudável, gerando apatia, auto desvalorização, insegurança e, consequentemente, leva o idoso ao isolamento social.

Em consequência do grande número de problemas com mobilidade, muitos projetos de cadeiras de rodas inteligentes (CRI) têm sido criados ao longo dos anos (SIMPSON, 2005). De acordo com (BRAGA, 2011), pode-se definir que uma CRI é um dispositivo robótico construído a partir de uma cadeira de rodas motorizadas, com sistemas de sensores, atuadores e capacidade de processamento. Também, uma CRI terá que ter navegação autônoma, planejamento autônomo, interface de interação homem-máquina, comportamento semi autônomo como desvio de obstáculos, e comportamento colaborativo e cooperativo. Assim, as CRI's podem ser uma boa solução para o desafio de assistir na mobilidade e capacidade de interação com o ambiente de pessoas com severas incapacidades e que não conseguem usar cadeiras motorizadas usuais em suas atividades diárias.

No entanto, mesmo com a grande quantidade de projetos sendo feitos no contexto das CRI, o foco de quase a totalidade destes projetos foi somente o desenvolvimento da tecnologia, tanto software como hardware, não se aprofundando ou se preocupando com as questões do design da cadeira, não havendo o entendimento da relação do usuário com o objeto.

Esse artigo tem por objetivo direcionar o design de uma cadeira de rodas inteligente fazendo o levantamento de requisitos deste tipo de equipamento, levando em conta a tecnologia, design e acessibilidade e apresentar uma abordagem metodológica no desenvolvimento deste tipo de produto. Também, acredita-se que com os métodos de prototipagem rápida juntamente com a modelagem da CRI em CADS convencionais pode-se chegar a estruturas formais com melhores soluções em relação ao design, principalmente devido ao fato desses tipos de produto contarem com inúmeros equipamentos. 


\section{REVISÃO BIBLIOGRÁFICA}

Nos últimos anos, muitas CRI's foram desenvolvidas e um grande número de projetos científicos na área se iniciou. Porém, a maioria deles não se preocupava com ergonomia ou antropometria, somente com o funcionamento sem levar em consideração o conforto e facilidade. Somente em 2009, mais de 90 publicações referenciadas a cadeira de rodas inteligentes foram encontradas no IEEE (Institute of Electrical and Electronics Engineers, Xplore Digital Library).

As primeiras propostas de uso de cadeira de rodas autônomas por deficientes físicos começam em 1986 (MADARASZ, 1986). Eles propuseram uma cadeira de rodas equipada com um microcomputador, câmera digital e sensor de distância ultrassônico. Objetivaram o desenvolvimento de um veículo capaz de operar sem a intervenção humana em ambientes populosos e com poucas ou nenhumas colisões com objetos ou pessoas. Já na pesquisa (HOYERANDHOLPER, 1993) apresenta-se a arquitetura de controle modular para uma cadeira omnidirecional. De acordo com eles, essa estrutura leva vantagem por dar mais inteligência local para cada unidade para produzir alta independência de outros módulos e um sistema de controle aberto. NavChair (Figura 1) é uma cadeira de rodas autônoma que tem algumas características interessantes como seguir a parede, desviar de obstáculos automaticamente e passar pela porta (LEVINE, 1999).

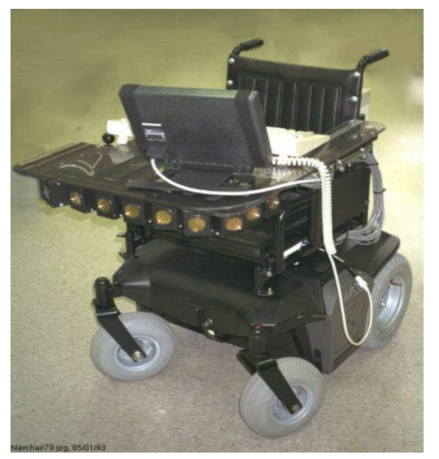

Figura 1 - NavChair

(Miller, 1998) desenvolveu a CRITin Man. Inicialmente esse sistema tinha 3 modos de operação: guiado por humanos com desvio de obstáculos, andar com comando, movimento para um ponto específico $(x, y)$. Depois, o projeto evoluiu para Tin Man II (figura 2) com a inclusão de novas funções, tal como backup, voltar ao ponto de origem, seguir as paredes e passagem por portas. Por incluir algumas das funções de Tin Man, o projeto Maid foi feito para navegar em duas situações particularmente cansativas e difíceis, como ambientes estreitos e desordenados, e grandes áreas aglomeradas (PRASSLER, 2001).

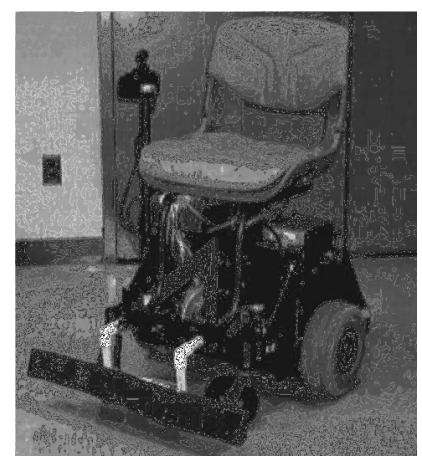

Figura 2 - Tin Man II 
(Wellman, 1994) propôs uma cadeira de rodas híbrida equipada com duas pernas além das quatro rodas, permitindo que a CRI pudesse subir os degraus e se mover por lugares com terreno acidentado. Alguns projetos apresentam soluções para pessoas com tetraplegia usando reconhecimento de expressões faciais como o meio principal de comando da cadeira. E também há CRI's que usam o "pensamento" como controle. Essa tecnologia tipicamente usa sensores para medir ondas eletromagnéticas do cérebro.

\section{DESIGN e ACESSIBILIDADE}

Um dos objetivos do Design Inclusivo é o da não discriminação e exclusão social por meio da construção de objetos, ambientes e serviços, isto é, respeitar a diferença pela utilização de todas as ajudas técnicas necessárias, mas sem, no entanto permitir que essas mesmas ajudas técnicas sejam por si só mecanismos de exclusão. $O$ design inclusivo é um design acessível e pode ser universal.

Segundo (Story, 1998), o design universal pode ser definido como o design de produtos ou ambientes que podem ser usados por pessoas de todas as idades ou portadores de deficiências. Certas soluções do Design Universal, não chamam a atenção tanto para as pessoas que desenvolveram como para o homem que as usa, pois na maioria das vezes eles são elaborados para facilitar uma tarefa ou necessidade individual. Ou seja, o design de produtos ou ambientes desenvolvidos a partir das considerações das necessidades de uma população diversificada, de homens, mulheres, crianças, idosos, pessoas baixas ou altas, portadoras de deficiências, atingem os objetivos do Design Universal, seja ele desenvolvido em ambientes escuros, ruidosos, úmidos e levando em conta a fadiga, isto, torna o Design Universal mais humano, pois um grupo maior de indivíduos é beneficiado.

\subsection{Tipos de design acessível}

Pesquisadores colocam o Design Universal como parte do Design Acessível, o mesmo que pode ser definido, como o desenvolvimento de produtos para serem usados por pessoas desabilitadas (incapazes). Muitos autores consideram que este tipo de produto acaba tornando o grupo segregado por ser portador de deficiências ou incapacidades, pois na maioria dos casos as soluções de acessibilidade são adicionadas após a finalização do projeto ou se faz um novo produto para satisfazer os requisitos de projeto. Normalmente, essas adições são realizadas após a constatação do fato ou após uma determinação da justiça. Exemplo disso são as rampas de acesso públicos, em complemento as escadas, barras de apoio ao longo de corredores, apoios em banheiros públicos, informações por meio de sinais táteis, sonoras etc.

Para Story (ibidem) O Design Universal é algumas vezes adaptável e transformado, mas sempre deve ser acessível. As relações entre os 4 tipos de Design podem ser vistos na Figura 3 


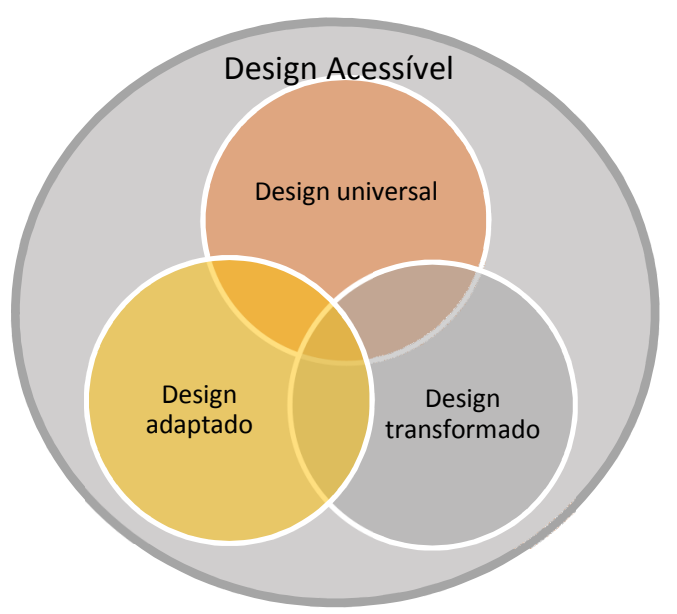

Figura 3-Tipos de Design Acessível

A figura 3 nos diz que as três abordagens de design estão interligadas entre si, no entanto são subordinados ao Design Acessível, ou seja, o propósito de cada um deles deve atender o requisito de acessibilidade, portanto nem todo design acessível é universal, e assim por diante.

Para Mullick e Steinfeld (1997) Design universal é diferente de Design acessível. Para os autores, o Design acessível são produtos que são utilizáveis por pessoas com inaptidões, já o Design universal significa produtos que são acessíveis e utilizáveis por todos, inclusive pessoas com inaptidões. Embora estas definições diferentes parecem ser simplesmente semânticas, eles na verdade têm diferenças. Design acessível tem uma tendência para separar instalações para pessoas com inaptidões, por exemplo, um jogo de rampa para fora ao lado de uma escada, ou um banheiro de deficiente do lado dos banheiros normais, ou uma cadeira de rodas customizada para determinada deficiência.

Dessa forma, a abordagem do Design universal provê uma solução que pode acomodar as pessoas com inaptidões como também o resto da população. As soluções prestam atenção às necessidades de pessoas mais velhas como também jovens, mulheres como também os homens, indivíduos canhotos como também os destros. Uma entrada que é projetada para ser "universal" não teria degraus. Uma cadeira de rodas com Design Universal também poderia acomodar as pessoas idosas ou com uma gama extensiva de limitações físicas.

\subsection{Design universal como alternativa crescente}

Durante os últimos anos nos países desenvolvidos tem surgido um interesse crescente em Design universal como uma alternativa para Design acessível. Isto tem acontecido por mudanças nos hábitos de compra, crescimento de mercados emergentes e melhoria da qualidade de vida. Alguns fatores são os seguintes:

- Aumento do número de deficientes;

- Maior poder aquisitivo entre a população com inaptidões;

- Desenvolvimento de um "mercado" de idosos;

- Reconhecimento das insuficiências dos serviços de assistências;

- Produtos e ambientes que não foram projetados para pessoas velhas e deficientes. 
Segundo o IBGE (web)

O crescimento da população de idosos, em números absolutos e relativos, é um fenômeno mundial e está ocorrendo a um nível sem precedentes. Em 1950, eram cerca de 204 milhões de idosos no mundo e, já em 1998, quase cinco décadas depois, este contingente alcançava 579 milhões de pessoas, um crescimento de quase 8 milhões de pessoas idosas por ano. As projeções indicam que, em 2050, a população idosa será de 1.900 milhões de pessoas. [...]. IBGE (web)

Por outro lado, o poder aquisitivo das pessoas com deficiências é significativo. Calcula-se que 36 milhões de pessoas inválidas no EUA gastam 40 bilhões dólares em produtos especiais. Eles fazem parte da vida da comunidade, trabalham e ganham mais dinheiro, eles estão começando a mostrar influência no desenvolvimento de produtos.

Segundo Steinfeld (1997) pesquisas de mercado do setor de tecnologia médica e de assistência médica, demonstraram que as pessoas não gostam do aparecimento de medicina e técnica nos produtos, eles preferem um design agradável, moderno. A pesquisa também mostrou que esses produtos são muito caros, difíceis de achar, difíceis de consertar e principalmente difíceis de usar. Talvez uma das limitações mais importantes seja que os consumidores os veem com uma linguagem estigmatizada "produtos especiais" o que promove um conceito negativo.

Crescentemente, os defensores de direitos de deficientes e profissionais de Design, perceberam que o conceito de Design acessível teria que ser repensado. Os produtos existentes no mercado foram projetados visando um tipo de consumidor, pessoas jovens, ativas e "normais". Para os pesquisadores, o desafio é mudar o modo como o designer pensa. É importante mencionar que o termo que Design universal não insinua que tudo neste mundo é completamente utilizável por todo o mundo. A proposta é, que o termo de "utilizável por todos" seja uma meta a ser alcançada e uma atitude humana por parte dos designers em lugar de uma regra rígida de design.

Os países menos desenvolvidos compartilham algumas das mesmas tendências como os países altamente desenvolvidos. Eles também estão sentindo um aumento de melhoria da qualidade de vida dos deficientes, assim como, o surgimento de mercados emergentes de consumidores da terceira idade. Nestes países, a assistência médica e produtos voltados para esses consumidores são muito mais cara e difícil de achar. Em alguns países como o Brasil está simplesmente fora do alcance da maioria das pessoas.

\subsection{Design universal vs design acessível}

Para Mullick e Steinfeld (ibidem) Design universal faz sentido como uma alternativa para Design acessível porque pode estar geralmente mais disponível o mais baixo custo que a criação de tecnologia de assistência. Nos países menos desenvolvidos, o design deveria criar a oportunidade para evitar produtos especiais e criar produtos universais.

Design universal também apresenta uma oportunidade para desenvolvimento econômico. A criação e produção de produtos que são mais fáceis de usar por todas as pessoas podem melhorar competitividade no mercado de exportação mundial. Segundo os autores, o primeiro passo para operacionalizar a filosofia de Design universal é projetar para uma gama maior das pessoas. 
Design acessível focaliza frequentemente as necessidades das pessoas que usam cadeiras de rodas ou têm deficiências visuais, o Design universal considera outras diferenças além das deficiências físicas. Isto inclui diferenças em força, habilidades intelectuais, percepções e valores. Por exemplo, Design universal de uma cadeira de rodas pensaria em um veículo que possa ser utilizado por pessoas sem deficiência, mas que precisa percorrer um espaço, um equipamento de reconhecimento de voz para quem tem deficiência nos membros superiores etc.

Design acessível tem frequentemente uma conotação médica ou institucional. Falta de estética atraente e uma linguagem moderna e sem apelo aos problemas do usuário. Os produtos universais mais divulgados expressam frequentemente características de utilidade do produto ou ambiente, assim como, qualidade e estética forte.

\subsection{Design universal para uma cadeira de rodas inteligente}

Segundo Story (ibidem) no Centro de Design Universal, da Universidade da Carolina do Norte, foram desenvolvidos os sete princípios do Design Universal para guiar o desenvolvimento de produtos e ambientes.

A seguir são mostrados os sete princípios relacionados ao desenvolvimento de uma cadeira de rodas.

\section{Princípio - Uso Equitativo}

A CRI deve ser utilizável e de custo razoável para pessoas com habilidades diversas; Deverá ter os mesmos significados de uso para todos os usuários, idêntico quanto possível, equivalente quando não possível; Prover uma carcaça para oferecer segurança e proteção de forma igualmente disponível para todos os usuários; Possuir um design atraente para todos os usuários.

\section{Princípio - Uso Flexível}

A CRI deve acomodar uma grande faixa de pessoas com diversas preferências e habilidades; Prover escolhas na forma de utilização, já seja por comando de voz, joystick etc.; Permitir a utilização para destros e canhotos; Facilitar a precisão e acuidade do usuário.

\section{Princípio - Uso simples e intuitivo}

O uso da CRI deverá ser de fácil compreensão, independente da experiência do usuário; Eliminar a complexidade desnecessária; Ser coerente com as expectativas e intuições do usuário; Acomodar uma ampla faixa de habilidades; Organizar as informações de forma compatível com sua importância; Providenciar respostas efetivas e sem demora durante e após o término da locomoção.

\section{Principio - Informação de Fácil Percepção}

O painel da CRI deve comunicar a informação necessária efetivamente para o usuário, independentemente das condições do ambiente ou das habilidades sensoriais do usuário: Usar diferentes formas (pictórico, verbal, táctil e sonoro) para apresentação das informações do painel ou no ambiente interno do veículo; Maximizar a legibilidade da informação essencial; Diferenciar elementos de forma a poderem ser descritos (isto e, tornar mais fácil dar informações ou direções); Prever 
compatibilidade com uma variedade de técnicas ou avisos usados por pessoas com limitações sensoriais.

\section{5o Princípio - Tolerância ao erro}

A CRI deve minimizar riscos e consequências adversas de ações acidentais ou não acidentais; Organizar os elementos de entrada e saída da CRI e as informações no painel para minimizar riscos erros; os elementos mais usados mais acessíveis; elementos de riscos ou perigosos eliminados, isolados ou protegidos; Possuir avisos de riscos e de erro; Possuir cinto de segurança, sistema simples de direção.

\section{Princípio - Baixo esforço físico}

A CRI deve ser usada de forma eficiente e confortavelmente e com o mínimo de esforço como não acontece nas cadeiras normais; Permitir ao usuário manter uma posição corporal neutra; Usar moderadas forças na operação; Minimizar ações repetitivas; Minimizar a sustentação de um esforço físico.

\section{Princípio - Dimensão e espaço para aproximação ao uso do produto}

A CRI deve ter dimensão e espaço apropriados para o acesso, a manipulação e uso independentemente do tamanho do corpo do usuário, da postura ou mobilidade; Colocar os elementos importantes no campo visual dos usuários sentados; Fazer com que o alcance de todos os componentes seja confortável para qualquer usuário sentado; Acomodar variações da dimensão dos controles.

\subsection{Ergonomia}

O objetivo da cadeira de rodas é compensar a incapacidade total ou parcial de andar, porém soluções de produtos de sucesso são baseadas nos usuários e na compreensão do ambiente que esse irá ser utilizado. Embora primariamente a cadeira de rodas seja um dispositivo de apoio destinado a fornecer mobilidade é também uma cadeira e como tal deve ser ergonomicamente projetada para fornecer conforto. BRANDT e SAMUELSSON (2009)

As cadeiras de rodas de propulsão manual proporcionam independência e mobilidade para os seus usuários, no entanto são causadoras de inúmeros problemas nos membros superiores devido ao uso excessivo de força nessa musculatura. ASKARI et al., (2013).

A usabilidade de produtos pode ser aperfeiçoada por meio de atributos conhecidos com a probabilidade de beneficiar os usuários em um contexto particular de uso. De modo a definir o grau de usabilidade alcançado é relevante mensurar o desempenho e satisfação dos usuários no momento de interação com o produto. A medição de usabilidade é particularmente importante para visualizar a complexidade das interações entre o usuário, os objetivos, as características da tarefa e os outros elementos do contexto de uso. Um determinado produto pode ter índices significativamente diferentes de usabilidade quando utilizados em diversos tipos de contextos.

Segundo, a ISO 9241-11 (1998), que define usabilidade como: a medida na qual um produto pode ser usado por usuários específicos para alcançar objetivos específicos com eficácia, eficiência e satisfação em um contexto específico de uso. 
Para a melhor compreensão desta última definição, cabe esclarecer o significado dos seguintes termos de acordo com Jordan (1998):

- Eficácia: se refere à extensão na qual uma meta é alcançada ou uma tarefa é realizada.

- Eficiência: se refere à quantidade de esforço requerido para se atingir uma meta. Quanto menos esforço, maior é a eficiência.

- Satisfação: se refere ao nível de conforto que os usuários sentem quando utilizam um produto e também ao nível de aceitação do produto pelos usuários para atingir as suas metas.

A definição da ISO 9241-11 (1998) deixa claro que a usabilidade não é uma propriedade intrínseca do produto isoladamente. Depende do seu "contexto específico de uso": quem está utilizando o produto; para qual finalidade; o tipo específico de ambiente.

Tentando identificar esses princípios de usabilidade no produto cadeira de rodas se faz necessário compreender os aspectos fisiológicos nos quais seus usuários estão sujeitos a sofrer com a utilização contínua do produto.

Muitos usuários de cadeira de rodas sofrem de dores nas costas e pescoço, movimentos restritivos juntamente com úlceras de pressão e desconforto são outras complicações associadas por estarem sentados por longos períodos. BRANDT e SAMUELSSON (2009)

A utilização da cadeira de rodas inclui propulsão com os membros superiores, iniciar, parar e mudanças de direção. Essa atividade de ação repetitiva dos braços exige um equilíbrio entre força muscular e resistência. $O$ paraplégico que possui menos controle do tronco pode levar a um maior esforço para os membros superiores. UZUN et al., (2011)

\section{REQUISITOS TECNOLOGICOS PARA CADEIRA INTELIGENTE}

Entende-se que para ser inteligente, uma cadeira de rodas elétrica precisa entender o que acontece ao seu redor, planejar suas próximas ações e reagir de acordo com as mudanças no ambiente, comandos e objetivos. Para que isto aconteça devem ser atendidos alguns requisitos tecnológicos.

Para permitir que uma cadeira de rodas tenha as funções necessárias de resposta, recomenda-se um hardware genérico e flexível o suficiente para que funcione com a maioria das cadeiras elétricas.

Podem-se dividir os requisitos de Hardware em três: entrada do usuário (joystick tradicional, USB joystick, teclado, microfone, câmera), sensores (ultrassom, encoder, webcam, infravermelho) e outros dispositivos (placa de comando e aquisição de dados, módulo de potência, unidade de processamento central - notebook).

\section{Entrada do usuário:}

- Joystick tradicional. Embora seja comum em cadeiras motorizadas, não é o suficiente para garantir a mobilidade de pessoas com incapacidades mais severas;

- Joystick de USB. Esse joystick é importante, pois vem com vários botões programáveis com funções de um nível mais alto;

- Teclado e tela sensível ao toque. Pode ser usado para configurar a cadeira.

Sensores: Para permitir a habilidade de desviar dos obstáculos, seguir as paredes, perceber desníveis no solo, é necessário: 
- 8 Sensor ultrassom;

- 12 Sensores infravermelhos;

- 2encoders;

- 1 webcam.

Outros dispositivos:

- Quadro de controle e aquisição de dados.

- Placa de interface. Esta é usada para obter as informações dos sensores e para enviar ao modulo de energia como e quando controlar os motores. Esse quadro é conectado a cadeira via USB.

- Módulo de potência. Converte comando de controle em sinal de potência que move a cadeira.

- Notebook comercial. Para rodar o software de controle em alto nível da CRI.

\subsection{Método proposto}

Considerando que o desenvolvimento de uma CRI com abordagem universal é um projeto de alta complexidade, foi adotado o método conhecido como Espiral de Projeto que é amplamente usado em projetos de alta complexidade e com equipes multidisciplinares, pois possuem sistemas complexos com alto grau de interdependência em que é difícil calcular os fatores simultaneamente. Desta forma, os itens de desenvolvimento são divididos em grupos dispostos de forma espiral, sendo que em que cada volta o desenvolvimento é revisado e aprofundado.

A Figura 4 mostra o modelo de projeto espiral para a CRI.

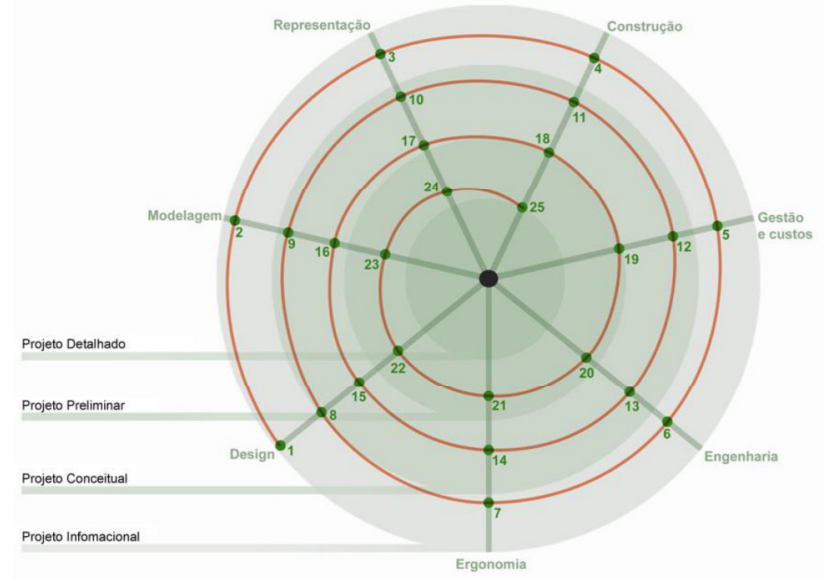

Figura 4 - Espiral do projeto da CRI

Fonte: "Elaborado pelo autor, com base na pesquisa realizada".

Segundo Hollister (2010), o desenvolvimento é dividido em quatro fases, a seguir a descrição relacionada ao desenvolvimento da CRI:

Projeto informacional- Define o objetivo da CRI e a lista de requisitos (tecnológicos, ergonômicos e de design) em ordem decrescente de importância.

Projeto conceitual- Esta fase determina se a CRI descrita no projeto informacional é factível e se os requisitos terão de ser modificados para que se consiga projetar a CRI com sucesso. É a parte mais criativa do processo de forma que a equipe de projeto não limite a criatividade, tentando gerar alternativas que apenas cumpram os requisitos. 
Projeto Preliminar- Esta etapa determina os detalhes tecnológicos e de design da CRI e de como serão alcançados os resultados do projeto conceitual.

Projeto Detalhado- Esta fase é relativa à produção documentos do projeto. Nesta fase serão feitos os desenhos finais da CRI, desenhos de construção e especificações.

\subsection{Resultado preliminar}

A seguir a Figura 5 mostra alguns detalhes da modelagem, que é o resultado do primeiro ciclo do modelo espiral da Figura 3. Considerando que as etapas realizadas até o momento foram: 1 . Desenvolvimento da lista de requisitos de projeto; 2 . Revisão de modelos de CRIs; 3. Representação gráfica 2D; 4. Análise de estrutura; 5. Análise de custo; 6 . Verificação da engenharia; 7. Análise de ergonomia e usabilidade; 8. Geração de alternativas; 9 . Modelagem onde está inclusa a modelagem que dará suporte para representação das alternativas.

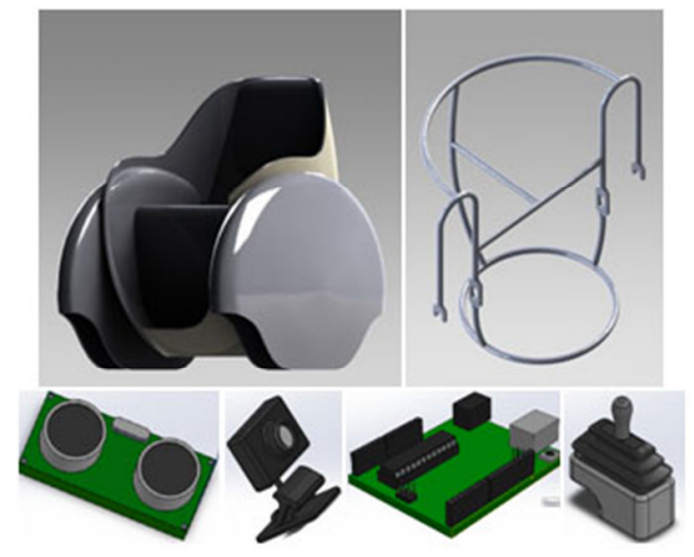

Figura 5 - Modelagem de uma alternativa da CRI e seus componentes

Fonte: "Elaborado pelo autor, com base na pesquisa realizada".

O modelo da estrutura de uma alternativa de solução de uma cadeira e dos componentes: Sensor ultrassônico, webcam, Joystick e placa de controle. Uma das configurações para adequar o usuário.

\section{Considerações Finais}

$\mathrm{O}$ artigo apresentou o resumo do projeto de uma cadeira de rodas inteligente dentro de uma abordagem de design universal e multidisciplinar com a parceria do "omitido revisão cega". O projeto está completado na primeira fase do modelo espiral e com previsão de realização das próximas etapas.

Foi apresentada uma revisão bibliográfica de cadeira de rodas inteligentes, design universal e os resultados preliminares do desenvolvimento da cadeira mostrando que se trata de um projeto de alta complexidade e alto valor social.

\section{REFERÊNCIAS}

BRAGA, Ram; PETRY, M; REIS LP; MOREIRA, Ap. IntellWheels: Modular Development platform for intelligent wheelchairs. J Rehabil Res Dev. 2011; 48(9):1061-76. DOI: 10.1682/ JRRD.2010.08.0139. 
BRANDT, Åseand SAMUELSSON, K. Wheel chair Intervention: Principles and Practice. I. Soderback (ed.), International Handbook of Occupational Therapy Interventions, (c) Springer Science Business Media, LLC 2009. DOI: 10.1007/978-0-387-75424-6_8.

HOLLISTER, Stephen M. The Design Spiral for Computer-Aided Design.< disponível em: http//www.newavesys.com/spiral.htm> . Acesso em 15 de maio de 2010.

HOYER, H; HÖLPER, R. Open control architecture for an intelligent omnidirectional wheelchair. Proceedings of the 1st TIDE Congress; 1993 Apr 6-7; Brussels, Belgium, Amsterdam (the Netherlands): IOS Press; 1993. p. 93-97.

IBGE http://www.ibge.gov.br/home/presidencia/noticias/25072002pidoso.shtm. Acesso em 01 jan. 2014.

ISO (1998). ISO 9241-11: Ergonomic requirements for office work with visual display terminals (VDTs).Part 11Guidelines for specifying and measuring usability. Genève: International Organization for Standardization.

JORDAN, P.W. An Introduction to Usability. London: Tylor\&Francis.1998.

LEVINE, SP; BELL, DA; JAROS, LA; SIMPSON, RC; KOREN, Y; BORENSTEIN, J. The NavChair Assistive Wheelchair Navigation System. IEEE Trans Rehabil Eng. 1999; 7(4):443-51. [PMID: 10609632] DOI: 10.1109/86.808948

MADARASZ, R; HEINY, L; CROMP, R; MAZUR, NM. The design of na autonomous vehicle for the disabled. IEEE J Robot Autom. 1986; 2(3):117-26.

MILLER, D. Assistive robotics: An overview. Lect Notes Comput Sei. 1998; 1458:126-36. DOI: $10.1007 / \mathrm{BFb} 0055975$

MULLICK, A; STEINFELD, Edward. Universal Design. What it is and isn't. Innovation Spring, 14-17 University of Buffalo. NY. 1997.

PRASSLER, E; SCHOLZ, J; FIORINI, P. A robotic wheelchair for crowded public environment. IEEE Robot Autom. 2001; 8(1):38-45. DOI:10.1109/100.924358

SIMPSON, Rc. Smart wheelchairs: A literature review. J RehabilRs Dev. 2005;423-36. [PMID: 16320139] DOI:10.1682/JRRD.2004.08.0101

STEINFELD, Edward. How does an idea spread \& grow. Innovation Spring, 19-24 University of Buffalo. NY. 1997.

STORY, Follette Molly; MUELLER L. James; MACE L. Ronald. The Universal Design File: Designing for People of All Ages and Abilities. NC State University Published by the Center for Universal Design 1998.

UZUN S; POURMOGHADDAM A; HIERONYMUS M; THRASHER T. A. Evaluation of muscle fatigue of wheelchair basketball players with spinal cord injury using recurrence quantification analysis of surface EMG. Eur J ApplPhysiol (2012) 112:38473857. DOI 10.1007/s00421-012-2358-0

WELLMAN, P; KROVI, V; KUMAR, V. An adaptive mobility system for the disabled. Proceedings of the IEEE International Conferences on Robotics and Automation; 1994 May 8-13; San Diego, CA. Los Alamitos (CA): IEEE; 1994. p. 2006-11. 